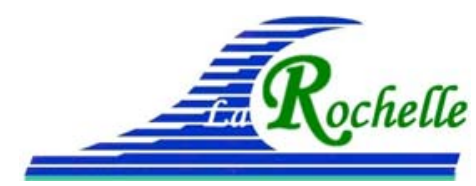

XV $V^{\text {èmes }}$ Journées Nationales Génie Côtier - Génie Civil

La Rochelle, 29 au 31 mai 2018

DOI:10.5150/jngcgc.2018.081

(c) Editions Paralia CFL

disponible en ligne - http://www.paralia.fr - available online

\title{
Habitats marins et estuariens de l'estuaire de la Gironde : bilan des connaissances et cartographie NATURA 2000
}

\section{Pierre-Guy SAURIAU ${ }^{1}$, Hugues BLANCHET ${ }^{2}$}

1. LIENSs, CNRS, Université de la Rochelle, UMR 7266 CNRS - ULR, 2 rue Olympe de Gouges, 17000 La Rochelle, France.

pierre-guy.sauriau@univ-lr.fr

2. EPOC, UMR 5805, Université de Bordeaux, CNRS, Station Marine d'Arcachon, Observatoire Aquitain des Sciences de l'Univers, 2 rue du Pr Jolyet, 33120 Arcachon, France.

hugues.blanchet@epoc.u-bordeaux.fr

\section{Résumé :}

L'estuaire de la Gironde est connu pour l'ampleur de son domaine estuarien, son caractère hyper turbide et une dynamique spatio-temporelle des échanges eau-sédiment très complexe. Les habitats marins et estuariens qu'il héberge sont connus et décrits sur le plan des espèces et des communautés par plus de quatre décennies de travaux d'écologie benthique qualitative et quantitative. Ces approches biocénotiques ont été complétées dans les années récentes par des approches cartographiques (projet CARTHAM), en se basant pour l'essentiel sur des cartographies biosédimentaires préexistantes et la prise en compte de données stationnelles dans la répartition des espèces. La cartographie des habitats intertidaux et subtidaux ainsi proposée était cependant lacunaire, sans prise en compte réelle de la diversité des habitats du domaine rocheux estuarien ou certaines particularités des estrans meubles sablo-vaseux. Est ici proposée une mise à jour de cette cartographie qui liste l'essentiel des habitats nouvellement répertoriés. Elle trouvera son utilité dans un contexte "amélioration des connaissances" de la gestion Natura 2000 de cet écosystème pour les gestionnaires du patrimoine naturel comme le Parc naturel marin de l'estuaire de la Gironde et de la mer des Pertuis.

Mots-clés :

Natura 2000, Estuaire, SIG, Cartographie, EUNIS, Parc naturel marin, Connaissances, Patrimoine. 


\section{Thème 6 - Gestion durable des zones littorales et estuariennes}

\section{Introduction}

L'estuaire de la Gironde est connu pour ses paysages alternant vasières, schorres, prés salés, îles d'estuaires, conches sableuses, coteaux calcaires bordés de carrelets et surmontés de vignobles mondialement renommés, ses pêcheries estuariennes et ses activités portuaires. Il joue cependant un rôle emblématique dans la conservation d'espèce endémique ou en danger d'extinction, comme l'angélique des estuaires Angelica heterocarpa (QUENNESON \& CASTAGNÉ, 2012) ou l'esturgeon Acipenser sturio, dernière population de l'ouest-européen (ACOLAS et al., 2017). Le domaine estuarien girondin s'étend sur plus de $165 \mathrm{~km}$, de l'embouchure (Pointe de Grave Pointe de Suzac) à l'amont de Bordeaux (ALLEN et al., 1974). Il héberge un système bouchon vaseux - crème de vase mais l'estuaire de la Gironde est décrit comme hyper turbide avec une dynamique spatio-temporelle complexe des échanges eau-sédiment (SOTTOLICHIO \& CASTAING, 1999). Tant les mesures ponctuelles que les mesures par télédétection (FROIDEFOND et al., 1998, DOXARAN et al., 2009) associées aux approches de modélisation confirment une localisation des maxima de turbidité sensiblement entre l'amont de Bordeaux en étiage et les environs de la Pointe de Grave en crue moyenne. En fortes crues, le bouchon vaseux est naturellement expulsé de l'estuaire et atteint alors le plateau continental (FROIDEFOND et al., 1998) avant de se reconstituer par les apports fluviaux. Cette dynamique sédimentaire associée à la géomorphologie particulière de l'estuaire, explique la présence généralisée de substrats meubles vaseux et de leurs habitats associés tout le long du chenal et des deux rives de l'estuaire. En rive droite cependant, entre Saint-Palais-sur-Mer et Talmont-sur-Gironde, les substrats rocheux liés aux falaises calcaires crayeuses du Crétacé Supérieur (Maestrichtien) créent une alternance de promontoires, conches, criques et schorres herbacés. A l'embouchure, en aval de la Pointe de Grave, c'est le domaine euhalin où dominent les sédiments sableux et granulaires. Des singularités géomorphologiques y apparaissent comme les îles sableuses, le platier rocheux de Cordouan (calcaire Eocène Moyen Lutécien) et la baie de Bonne Anse en arrière de la flèche littorale "Pointe de la Coubre" (FAÇON, 1965). Tous ces éléments hydro-géomorphologiques ont été synthétisés puis combinés à une double expertise faune/flore s'étalant sur près d'une quarantaine d'années (BACHELET, 1979, ESTÈVE \& LAHONDÈRE, 1979, LAHONDÈRE, 2002, BLANCHET et al., 2014) et sédimentaire (HAMDI et al., 2010) pour dresser en 2011 un premier bilan des habitats Natura 2000 de l'estuaire de la Gironde (programme CARTHAM de l'Agence Française pour la Biodiversité, ex AAMP). Depuis lors de nouvelles observations faune/flore ont permis d'affiner ces connaissances et de combler des lacunes sur des habitats très localisés mais révélateurs $\mathrm{du}$ fonctionnement biologique de l'estuaire. L'objectif est ici d'en dresser un nouveau bilan potentiellement utile aux gestionnaires et opérateurs Natura 2000 de l'estuaire. 


\section{XVèmes Journées Nationales Génie Côtier - Génie Civil La Rochelle, 29 au 31 mai 2018}

\section{Matériel et méthodes}

Initiées il y a plus de 40 ans (BOUCHET \& LISSALDE, 1976, BACHELET, 1979), les études sur le benthos de substrats meubles de la Gironde sont aujourd'hui principalement liées aux suivis pluri-décennaux de l'impact de la centrale nucléaire du Blayais (BACHELET \& LECONTE, 2016) ou ceux des suivis de la surveillance DCE de l'état écologique des eaux de transition (BLANCHET et al., 2014). Des approches naturalistes viennent les compléter pour les estrans intertidaux sableux en rive droite de l'estuaire avec la description de plus de 120 espèces (VOLLETTE \& THIRION, 2011b; a; 2015) ou sur le platier rocheux de Cordouan (GUYONNEAU, 2010, PIGEOT, 2013). Les inventaires récents du SMIDEST sur la végétation des schorres et berges de l'estuaire (GEREA, 2016a; b) permettent une caractérisation plus poussée des habitats végétalisés. De même, les mosaïques de macroalgues de la rive droite entre SaintPalais-sur-Mer et la Pointe de Suzac ont fait l'objet d'un bilan floristique, avec près de 60 espèces (VOLLETTE et al., 2016) permettant de positionner un suivi de la surveillance DCE en eau de transition sur cet indicateur (SAURIAU, données non publiées). La cartographie utilise la typologie EUNIS élaborée par l'Agence Européenne de l'Environnement (http://eunis.eea.europa.eu/habitats.jsp), complétée par la typologie MNHN version 2 (MICHEZ et al., 2015) élaborée spécifiquement pour les habitats benthiques de la façade Manche-Atlantique. L'intitulé de chaque habitat reprend celui de la typologie MNHN ou, sauf exceptions, celui traduit par BAJJOUK et al. (2015) de la typologie EUNIS. Les superficies sont issues de la cartographie des habitats marins CARTHAM (http://cartographie.aires-marines.fr/?q=node/43) et des cartes de végétation (GEREA, 2016a; b).

\section{Résultats}

\subsection{Estuaire externe : aval Pointe de Grave/Royan jusqu'à Bonne Anse/Cordouan}

Le domaine intertidal de Bonne Anse présente de larges vasières intertidales estuariennes dominées par les Polychètes/Bivalves (A2.3 - A2.31) dont l'habitat à Scrobicularia plana et Hediste diversicolor (A2.313) décrit par ESTĖVE \& LAHONDÈRE (1979). De nos jours, l'envasement plus marqué permet le développement d'une pêcherie à Ruditapes philippinarum très productive (HENNACHE, CREAA, communication personnelle). Vers la côte, la slikke se poursuit par un schorre (A2.5) où se succèdent, selon l'hypsométrie et les espèces végétales, les habitats A2.51 à A2.55 ainsi que les habitats de laisses de mer (B1.1). Ce schorre présente de nos jours (GEREA, 2016a; b) des mosaïques singulières de spartines pionnières (A2.55) et d'herbiers à Zostera noltei (A2.6111). Les premières observations d'herbiers à Zostera noltei à Bonne Anse sont assez récentes (LAHONDÈRE, 2002) en regard des observations régulièrement réalisées sur ce site depuis le premier bilan botanique (ESTĖVE \& LAHONDĖRE, 1979). Vers le chenal, les estrans de sables 


\section{Thème 6 - Gestion durable des zones littorales et estuariennes}

envasés intertidaux (A2.24) sont caractérisés par 1'habitat à Cerastoderma edule et Arenicola marina (A2.242) décrit par ESTÈVE \& LAHONDÈRE (1979). Les habitats typiques des plages sableuses A2.211, A2.22 et A2.23 sont en revanche présents dans la partie orientale de la baie, à proximité de son embouchure et se poursuivent vers Royan. Dans la Grande Conche de Royan, sont décrits par VOLLETTE \& THIRION (2011a) d'abondantes populations d'Arenicola marina dans des sables fins envasés à Cerastoderma edule et Polychètes (A2.242). Cet habitat est également présent dans la conche de St-Palais. Côté, rive droite, le domaine intertidal de la Pointe de Grave vers Soulac n'est que peu décrit mais les sables intertidaux mobiles propres (A2.22) à Donax vittatus y sont connus (BOUCHET \& LISSALDE, 1976). Viennent les compléter les sables fins et grossiers intertidaux des bas de plage (A2.22 et A2.23) jusqu'aux sables à Talitres (A2.211) en pied du domaine dunaire pour autant que celui-ci n'est pas ni endigué ni garni de parapets bétonnés (J3.53).

Le domaine intertidal rocheux rive gauche présente, comme à Saint-Palais, une succession remarquable d'habitats rocheux dont la base est ennoyée par les sables des conches. Cette succession est d'autant plus remarquable qu'elle s'effectue en quelques mètres. Les sables infralittoraux en pied de falaises relèvent, selon les variations de granulométrie, des habitats A5.22, A5.23 ou A5.24. Ces sables permettent en bas de plage l'existence de récifs à hermelles Sabellaria alveolata (A2.71) bien développés et de taille décimétriques. Remis en suspension sous les mouvements du ressac et des courants de marée, ils génèrent en pied de falaise un faciès rocheux d'abrasion azoïque puis un habitat à plaquages de Sabellaria alveolata (A1.2). Celui-ci se mélange avec la ceinture à Fucus serratus et algues rouges (A1.15). La mosaïque se complexifie ensuite avec l'apport d'huîtres Crassostrea gigas sur roche (A1.4) et de sable incrusté permettant un développement d'algues vertes (A2.82) ou la présence de moule Mytilus edulis (A1.326). Progressivement avec l'hypsométrie croissante et le changement dégressif de pente, la mosaïque se simplifie car les hermelles laissent la place aux huîtres et patelles (A1.11). Le médiolittoral supérieur de l'estran rocheux présente une rupture de pente colonisée par des balanes et patelles (A1.11) qui deviennent éparses (A1.1) dans les cuvettes (A1.41) avant de se terminer par les habitats de lichen du supralittoral avec la bande noire caractéristique de Hydropunctaria maura (B3.113). Les constructions urbaines de front de mer (J1) et les digues maritimes (J2.53) sont également impactées par les embruns. Sur les flancs extérieurs de la conche, l'estran est plus large en forme de plateau subhorizontal mais est parcouru par de grandes failles profondes avec un écartement parfois métrique. Ces failles hébergent les habitats de caves et grottes médiolittorales (A1.448) dont les parois et fondement toujours en eau ou humides sont typiques des grottes infralittorales (A3.715 et A3.716). Cet estran calcaire subhorizontal est lui aussi tout à fait remarquable puisqu'il présente un faciès "Lapiaz" issu de l'altération des roches calcaires du Maastrichtien par les actions chimiques de l'eau. Ce faciès présente une mosaïque de petites flaques médiolittorales à 


\section{XVèmes Journées Nationales Génie Côtier - Génie Civil La Rochelle, 29 au 31 mai 2018}

patelles, balanes et Actinia equina (A1.41), de roches à faible couverture macrobiotique (A1.1) ou de roches couvertes de balanes (A1.11). Le massif rocheux des Pierrières plus en amont est caractérisé par une singularité géologique avec la superposition en discordance (lacune sédimentaire de 20 millions d'années) de calcaires Eocène sur une base de calcaires Crétacé Maastrichtien (CHARLES, 2012). L'érosion de ces calcaires Maastrichtien a aussi généré de large failles et grottes médiolittorales (A1.448). Le platier héberge l'habitat de plaquages à Sabellaria alveolata (A1.2) générant par endroit le développement de petits récifs à Sabellaria alveolata (A2.71). En arrière de la zone de transport et maintien du sable suspension favorables aux hermelles, le platier est composé jusqu'au pied des falaises de mosaïques complexes de ceinture algale de Fucales (A1.12 et A1.15), de sables mobiles (A2.2), de roches et blocs à faibles couvertures macrobiotiques (A1.1), de roches et blocs à huîtres (A1.4) et de cuvettes (A1.41). La conche sableuse plus en aval présente des habitats intertidaux de sédiments grossiers intertidaux en milieux à salinité variable (A2.12) et des récifs d'huîtres intertidaux sur substrats meubles (A2.7). Le front de mer rocheux de St-Palais, à proximité du puits de l'Auture, outre son paysage de pêcherie au carrelet, présente un développement remarquable de moulière intertidale sur roche Mytilus edulis (A1.111) sur la partie plane du médiolittoral moyen alors que le médiolittoral inférieur et l'infralittoral exondable sont colonisés par de large plaquages et récifs à Sabellaria alveolata (A2.71). Ces moulières dans l'infralittoral supportent des conditions de salinité variable (A3.361). Le front de mer rocheux de St-Palais, en direction de la Grande Côte de l'ouest, présente aussi un paysage remarquable avec une vaste plage sableuse (A2.22) venant jusqu'en pied de falaise calcaire où se développe une mosaïque d'habitats à algues vertes sur sable (A2.82) et moule Mytilus edulis sur roche (A1.111). Du fait de la violence des courants et du rôle abrasif du sable, l'habitat huître ne se développe pas aux bénéfices de l'habitat de patelles et balanes (A1.11). La structure du faciès de "Lapiaz" sur les platiers subhorizontaux du médiolittoral supérieur permet alors à de petites cuvettes médiolittorales de se développer (A1.41) avant que la ceinture à lichens (B3.11) avec Hydropunctaria maura (B3.113) ne prenne le relai y compris sur la strate Eocène de calcaires gréseux plus clairs. Les observations faunistiques de (VOLLETTE \& THIRION, 2011b; 2015) sur les estrans rocheux entre Saint-Palais-surMer et la pointe de Suzac confirment la présence, parmi plus d'une centaine d'espèces animales observées, d'espèces communes des habitats de ces roches intertidales (cirripèdes, huîtres, moules, patelles, mollusques perforants avec cryptofaune associée) et potentiellement un habitat à Modiolus barbatus en bas d'estrans. Ces estrans rocheux hébergent également 30 à 40 espèces de macroalgues par site pour un total d'environ 60 espèces connues sur l'estuaire (VOLLETTE et al., 2016).

Le domaine subtidal de l'estuaire externe est dominé par les substrats de sables grossiers et graviers infralittoraux (A5.13), les sables fins propres ou envasés sublittoraux (A5.23) parmi lesquels les sables dunaires à Spisula solida ou sables 


\section{Thème 6 - Gestion durable des zones littorales et estuariennes}

mobiles propres infralittoraux à faune éparse (A5.231 et les sables fins infralittoraux à Chamelea striatula - Mactra stultorum décrits par BOUCHET \& LISSALDE (1976). Des roches et blocs infralittoraux (A3) liés aux roches calcaires Eocène Moyen (A3.2 mode exposé, A3.3 mode abrité) émergent de cet ennoiement sableux mais leurs faunes et flores sont inconnues à ce jour. Le platier rocheux de Cordouan relève du domaine médiolittoral et infralittoral où il est possible d'individualiser des mosaïques habitats sur la base des descriptions et relevés faune-flore de PIGEOT (2013) : cirripèdes, patelles et huîtres des roches et blocs médiolittoraux (A1.11), roches et blocs médiolittoraux à dominance animale (A1.2) associé à des bancs de sables découvrant (A2.22), habitats à dominances animales y compris de faune perforantes (A1.11) en mélange avec des habitats à dominances végétales à algues rouges sans Fucales (A1.12), cuvettes permanentes en milieu rocheux de la zone médiolittorale à Corallinales encroûtantes (A1.411) et, pour l'infralittoral, une zone à Laminaires clairsemées dominées par Saccorhiza polyschides (A3.122).

\subsection{Estuaire interne : Pointe de Grave/Pointe de Suzac jusqu'à Bordeaux}

Le domaine intertidal meuble (Figure 1, Tableau 1) se caractérise par de larges vasières estuariennes nues sans végétation dites slikke (A2.3) qui se distribuent largement, rive gauche, dans l'anse du Verdon jusqu'à Valeyrac et, rive droite, en petites vasières enclavées comme aux environs de Meschers-sur-Gironde, Talmont-sur-Gironde et Barzan. Ces vasières nues s'effilochent vers l'amont en d'étroites berges. Les neuf espèces caractéristiques de la slikke (Scrobicularia plana, Limecola balthica, Peringia ulvae, Hediste diversicolor, Streblospio shrubsolii, Heteromastus filiformis, Tubificoides heterochaetus, Corophium volutator et Cyathura carinata) permettent de décliner les habitats génériques A2.31 et A2.32 en habitats élémentaires selon l'hypsométrie et la dominance des espèces de Mollusques, Polychètes et Oligochètes. En superficie, ce sont les principaux habitats intertidaux de l'estuaire (Tableau 1). Le haut de ces vasières se transforme en un schorre qui abrite les habitats classiques à Angiospermes estuariens (A2.5). Cet habitat générique se décline selon l'hypsométrie et le degré halin en cortège d'espèces caractéristiques d'habitats élémentaires depuis les stades pionniers à Spartines jusqu'aux prés salés et roselières à scirpes et phragmites. Ceux-ci sont très généralement intriqués et disposés en mosaïques (GEREA, 2016a; b). La végétation du schorre est particulièrement bien développée en rive droite entre Barzan et Saint-Thomas-de-Conac (PK 60) et, rive gauche, en isolats comme à Talais près du Verdon. Ce schorre girondin est fortement mité par les mares de tonne $(\mathrm{C} 1.5$ et C1.6 selon leur permanence) dont certaines sont décrites pour héberger une faune invertébrée très diversifiée (LABAT \& SERRETTE, 2015). Localement peuvent également s'individualiser des habitats de galets et cailloutis intertidaux (A2.11) en pied d'enrochement. Plus vers l'amont, en domaine oligohalin, ces vasières s'effilochent en d'étroites berges avec une végétation qui épouse toutes les indentations du rivage. En 


\section{XVèmes Journées Nationales Génie Côtier - Génie Civil La Rochelle, 29 au 31 mai 2018}

domaines oligohalin et limnique, les roselières à phragmites (C3.21) sont bien développées. Les roselières à scirpes (C3.27) ne s'étendent que jusqu'au domaine mésohalin. En complément, le domaine intertidal des petites conches situées rive droite entre la Pointe de Suzac et l'aval de Meschers-sur-Gironde présente les habitats successifs des sables des hauts de plage à Talitres (A2.21 - A2.211), sables fins intertidaux dominés par les Polychètes/Amphipodes (A2.23) puis des sables envasés intertidaux dominés par les Polychètes/Bivalves (A2.24) tels Limecola balthica et Arenicola marina (A2.241) comme décrit par BOUCHET \& LISSALDE (1976). Cet habitat est en contact avec les sables envasés infralittoraux (A5.24) et les sables fins à moyens sublittoraux en milieu à salinité variable (A5.22) du chenal.

De nombreuses berges sont artificiellement enrochées, tant en milieu estuarien (J2.5) que marin (J2.53). Très localement, les substrats rocheux polyhalins soumis à dessalure comme entre Meschers-sur-Gironde et Talmont-sur-Gironde présentent des développements algaux, généralement de Fucales (A1.31 et A1.32). Les observations faunistiques de VOLLETTE \& THIRION (2011b) et floristiques de VOLLETTE et al. (2016) permettent de confirmer sur ces roches de l'intertidales à l'infralittoral exondable la présence de cirripèdes, huîtres, moules et patelles des roches et blocs médiolittoraux (A1.11), moulières intertidales sur roches et blocs (A1.111) et partiellement l'habitat à Modiolus barbatus ainsi que les divers habitats à fucales (A1.31 et A1.32). En surplomb, les falaises calcaires maritimes nues (GEREA, 2016a; b) et/ou végétalisées (LAHONDÈRE, 1996) sont bien représentées comme à Royan, St-Georges-de-Didonne, Meschers-sur-Gironde ou Talmont-sur-Gironde (B3.23 et B3.31).

Le domaine subtidal est très largement dominé par les habitats vaseux à salinités variables (A5.32). La zone d'influence maximale du bouchon vaseux - crème de vase $\mathrm{y}$ génère des habitats à vases fluides (A5.324) potentiellement situés dans les deux zones maximales de concentration en sédiments décrites par SOTTOLICHIO \& CASTAING (1999) entre les PK 50-60 et PK 0-40. De même, l'habitat élémentaire de sédiments envasés infralittoraux en milieu à salinité réduite définis par Limnodrilus hoffmeisteri, Tubifex tubifex et Gammarus spp. (A5.327) est potentiellement présent puisque les espèces Limnodrilus hoffmeisteri et Gammarus zaddachi sont très largement présentes dans l'estuaire (BACHELET \& LECONTE, 2016). L'habitat de sables fins à moyens sublittoraux en milieu à salinité variable (A5.22) vient compléter vers l'aval de l'estuaire interne l'habitat A5.32 précédent. Il est caractérisé par l'abondance des cortèges faunistiques vagiles décrit par SORBE (1981) avec Mesopodopsis slabberi et Neomysis integer, espèces qui caractérisent l'habitat élémentaire A5.223. L'habitat générique A5.22 se poursuit vers l'estuaire externe à l'aval de Royan par les sables fins infralittoraux (A5.23) connus en milieu marin. La coupure cartographique entre ces deux habitats reste arbitraire car fonction du régime halin mais reste cohérente avec la présence en rive droite de conches sableuses en aval de Meschers-sur-Gironde, elles même alimentées par ces sables infralittoraux. Quelques îlots de sédiments hétérogènes 


\section{Thème 6 - Gestion durable des zones littorales et estuariennes}

sont individualisables en milieu de chenal et relèvent de l'habitat générique A5.42 des sédiments hétérogènes sublittoraux en milieu à salinité variable. En complément, l'habitat générique de sables grossiers et graviers sublittoraux en milieux à salinité variable (A5.12) est présent dans l'estuaire dans la partie la plus concave de l'estuaire, rive gauche à Saint-Christoly-Médoc, là où très probablement les courants de marée sont les plus forts en bordure du chenal de navigation. En bordure de la zone intertidale sont décrits les sables envasés infralittoraux (A5.24) qui probablement héberge dans la partie aval de l'estuaire les sables fins légèrement envasés à Echinocardium cordatum et Ensis spp. (A5.241) puisque des tests d'Echinocardium fraichement morts s'échouent dans les conches sablo-vaseuses de la rive droite (SAURIAU, observation personnelle).

\section{Conclusions}

Cette cartographie de 2011 mise à jour illustre la diversité des habitats marins et estuariens de l'estuaire de la Gironde, hébergeant environ 240 espèces de la macrofaune et une soixantaine d'espèces de macroalgues. De remarquables mosaïques d'habitats comparables à ceux observés en estuaire de Seine (BAFFREAU et al., 2017) sont mieux individualisés en liaison avec l'hétérogénéité des substrats, comme par exemple les habitats de grottes marines intertidales, les herbiers de Zostera noltei en progression sur Bonne-Anse ou l'alternance de substrats rocheux/meubles en rive droite favorables à la présence de ceintures macroalgales. Ce bilan reste malgré tout provisoire et évolutif car dépendant de l'effort de prospection et des variations environnementales en cours et à venir.

Tableau 1. Habitats benthiques intertidaux de l'estuaire de la Gironde selon les typologies EUNIS 2012 et MNHN 2015. Superficie notée "présence" si surface non reportée et "-" si non individualisée sur la cartographie.

\begin{tabular}{|c|c|c|c|}
\hline Habitat & $\begin{array}{l}\text { Superficie } \\
\text { (ha) }\end{array}$ & $\begin{array}{l}\text { EUNIS } \\
2012\end{array}$ & $\begin{array}{l}\text { MNHN } \\
2015\end{array}$ \\
\hline $\begin{array}{l}\text { Falaises, corniches, rivages et îlots rocheux sans } \\
\quad \text { végétation } \\
\text { Falaises, corniches et rivages rocheux à Angiospermes }\end{array}$ & 248 & $\begin{array}{l}\text { B3.23 } \\
\text { B3.31 }\end{array}$ & - \\
\hline $\begin{array}{l}\text { Roches et blocs supralittoraux à lichens } \\
\text { Roches et blocs supralittoraux à lichens jaunes et gris } \\
\text { Roches et blocs supralittoraux à Hydropunctaria maura }\end{array}$ & présence & \begin{tabular}{l|l|} 
B3.11 \\
B3.111 \\
B3.113 \\
\end{tabular} & $\begin{array}{c}\text { R01 } \\
\text { R01.01 } \\
\text { R01.03 } \\
\end{array}$ \\
\hline $\begin{array}{l}\text { Berges artificielles enrochées } \\
\text { Digues en milieu marin }\end{array}$ & $\begin{array}{c}9,7 \\
1 \text { en linéaire }\end{array}$ & $\begin{array}{c}\mathrm{J} 2.5 \\
\mathrm{~J} 2.53\end{array}$ & $\begin{array}{l}- \\
-\end{array}$ \\
\hline $\begin{array}{l}\text { Roches et blocs du médiolittoral supérieur à très faible } \\
\text { couverture macrobiotique }\end{array}$ & présence & A1.1 & R04.01 \\
\hline Cirripèdes et patelles des roches et blocs médiolittoraux & présence & A1.11 & R03.01 \\
\hline Cirripèdes et huîtres des roches et blocs médiolittoraux & présence & A1.11 & R03.03 \\
\hline $\begin{array}{l}\text { Roches calcaires médiolittorales à Bivalves foreurs et } \\
\text { cryptofaune associée }\end{array}$ & présence & A1.11 & R03.06 \\
\hline Moulières intertidales sur roches et blocs & présence & A1.111 & P08.01 \\
\hline $\begin{array}{l}\text { Roches et blocs médiolittoraux à dominance d'algues } \\
\text { rouges }\end{array}$ & présence & A 1.12 & R02.04 \\
\hline
\end{tabular}




\section{XVèmes Journées Nationales Génie Côtier - Génie Civil La Rochelle, 29 au 31 mai 2018}

\begin{tabular}{|c|c|c|c|}
\hline $\begin{array}{l}\text { Fucales des roches et blocs du médiolittoral moyen } \\
\text { Fucales des roches et blocs du médiolittoral inférieur }\end{array}$ & présence & A 1.15 & $\begin{array}{l}\text { R02.02 } \\
\text { R02.03 }\end{array}$ \\
\hline $\begin{array}{l}\text { Roches et blocs médiolittoraux à dominance animale } \\
\begin{array}{l}\text { Plaquages de Sabellaria alveolata sur roches } \\
\text { médiolittorales }\end{array}\end{array}$ & 275 & $\begin{array}{l}\mathrm{A} 1.2 \\
\mathrm{~A} 1.2\end{array}$ & $\begin{array}{c}\text { R03 } \\
\text { R03.04 }\end{array}$ \\
\hline Fucales des roches et blocs du médiolittoral moyen & 2 & A1.31 & $\mathrm{R} 02.02$ \\
\hline $\begin{array}{l}\text { Roches et blocs intertidaux avec fucales en milieu à } \\
\text { salinité variable }\end{array}$ & 9 & A 1.32 & R06 \\
\hline $\begin{array}{l}\text { Roches et blocs intertidaux en milieu à salinité variable à } \\
\text { Fucus serratus et Mytilus edulis }\end{array}$ & présence & A1.326 & R06.06 \\
\hline Récifs d'huîtres intertidaux sur roches et blocs & 3 & $\mathrm{~A} 1.4$ & P10.01-02 \\
\hline $\begin{array}{l}\text { Cuvettes en milieu rocheux de la zone médiolittorale } \\
\text { Cuvettes en milieu rocheux de la zone médiolittorale à } \\
\text { Corallinales encroûtantes }\end{array}$ & présence & $\begin{array}{l}\text { A1.41 } \\
\text { A1.411 }\end{array}$ & $\begin{array}{c}\text { P18.02 } \\
\text { P18.02.01 }\end{array}$ \\
\hline $\begin{array}{l}\text { Grottes marines et surplombs intertidaux à faune } \\
\text { encroûtante } \\
\text { Grottes marines et surplombs intertidaux à faune éparse }\end{array}$ & présence & A1.448 & $\begin{array}{l}\mathrm{P} 20.01 .08 \\
\mathrm{P} 20.01 .09 \\
\end{array}$ \\
\hline Récifs à Sabellaria alveolata sur roches et blocs & présence & A2.71 & P12.01 \\
\hline $\begin{array}{l}\text { Roches et blocs infralittoraux en milieu à salinité variable } \\
\text { à Mytilus edulis }\end{array}$ & présence & A3.361 & R08.09.02.01 \\
\hline Bancs de Modiolus barbatus & présence & - & P09.03 \\
\hline Sables des hauts de plage à Talitres & 44 & A2.211 & M02.02 \\
\hline Galets et cailloutis intertidaux & 8 & A2.11 & M03.01 \\
\hline Laisse de mer à dessiccation lente & présence & $\mathrm{B} 2.12$ & M01 \\
\hline $\begin{array}{l}\text { Sédiments grossiers intertidaux en milieux à salinité } \\
\text { variable }\end{array}$ & présence & $\mathrm{A} 2.12$ & M03.03 \\
\hline $\begin{array}{l}\text { Sables intertidaux mobiles propres } \\
\text { Sable à Donax sp. }\end{array}$ & 80 & $\begin{array}{c}\mathrm{A} 2.22 \\
-\end{array}$ & $\begin{array}{c}\text { M04.01.01 } \\
\text { M04.01.01.03 }\end{array}$ \\
\hline $\begin{array}{lcccc}\begin{array}{l}\text { Sables fins intertidaux } \\
\text { Polychètes/Amphipodes }\end{array} & \text { dominés } & \text { par les } \\
\end{array}$ & 164 & $\mathrm{~A} 2.23$ & M04.02.01 \\
\hline $\begin{array}{l}\text { Sables envasés intertidaux } \\
\text { Polychètes/Bivalves }\end{array}$ & 57 & $\mathrm{~A} 2.24$ & M04.02.02 \\
\hline $\begin{array}{l}\text { Sables envasés intertidaux à Macoma balthica et Arenicola } \\
\text { marina }\end{array}$ & - & A2.241 & M04.02.02.01 \\
\hline $\begin{array}{l}\text { Sables envasés intertidaux à Cerastoderma edule et } \\
\text { Polychètes }\end{array}$ & - & A2.242 & M04.02.02.02 \\
\hline Vases intertidales estuariennes de la slikke & 4264 & A2.3 & M05.03 \\
\hline $\begin{array}{l}\text { Vases intertidales estuariennes dominées par les } \\
\text { Polychètes/Bivalves }\end{array}$ & 3734 & $\mathrm{~A} 2.31$ & M05.03.01.01 \\
\hline $\begin{array}{l}\text { Vases intertidales estuariennes dominées par les } \\
\text { Polychètes/Oligochètes }\end{array}$ & 530 & A 2.32 & M05.03.01.02 \\
\hline Vasières intertidales estuariennes du schorre & 1793 & $\mathrm{~A} 2.5$ & M05.02 \\
\hline ... haut schorre & 7 & $\mathrm{~A} 2.52$ & M05.02.01 \\
\hline$\ldots$ schorre moyen & - & $\mathrm{A} 2.53$ & M05.02.02 \\
\hline .... bas schorre & - & $\mathrm{A} 2.54$ & M05.02.03 \\
\hline Marais salés pionniers & 125 & $\mathrm{~A} 2.55$ & - \\
\hline Laisses des marais salés & - & $\mathrm{A} 2.51$ & - \\
\hline $\begin{array}{l}\text { Herbiers à Zostera noltei en milieu à salinité variable } \\
\text { Accumulation de débris de Zostera noltei ou autres } \\
\text { phanérogames en laisses de mer }\end{array}$ & $\begin{array}{l}196 \\
\text { présence en } \\
\text { linéaire }\end{array}$ & $\begin{array}{l}\mathrm{A} 2.611 \\
\mathrm{~B} 1.1\end{array}$ & $\begin{array}{l}\mathrm{P} 01.01 .02 \\
\mathrm{P} 01.01 .03\end{array}$ \\
\hline Récifs d'huîtres intertidaux sur substrats meubles & présence & A2.7 & P10.01.01 \\
\hline $\begin{array}{l}\text { Sédiments hétérogènes envasés intertidaux à algues vertes } \\
\text { ou rouges éphémères }\end{array}$ & présence & A2.82 & M06.02.03 \\
\hline Roselières à phragmites et scirpes & 565 & C3.21et C3.27 & - \\
\hline Lac de tonne & 126 & $\mathrm{C} 1.5$ et $\mathrm{C} 1.6$ & - \\
\hline
\end{tabular}




\section{Thème 6 - Gestion durable des zones littorales et estuariennes}

Tableau 2. Habitats benthiques subtidaux de l'estuaire de la Gironde selon les typologies EUNIS 2012 et MNHN 2015. Superficie notée "présence" si surface non reportée et "-" si non individualisée sur la cartographie.

\begin{tabular}{|c|c|c|c|}
\hline Habitat & $\begin{array}{l}\text { Superficie } \\
\text { (ha) }\end{array}$ & $\begin{array}{c}\text { EUNIS } \\
2012\end{array}$ & $\begin{array}{l}\text { MNHN } \\
2015\end{array}$ \\
\hline Roches et blocs infralittoraux & Présence & A3 & R08 \\
\hline $\begin{array}{l}\text { Zones à Laminaires clairsemées dominées par } \\
\text { Saccorhiza polyschides }\end{array}$ & - & A3.122 & R08.05.04 \\
\hline Roche infralittorale en mode exposé & 1309 & A 3.2 & - \\
\hline Roche infralittorale en mode abrité & 310 & A3.3 & - \\
\hline $\begin{array}{l}\text { Grottes marines et surplombs sublittoraux à } \\
\text { Spongiaires encroûtants }\end{array}$ & Présence & A3.715 & P20.02.05 \\
\hline $\begin{array}{l}\text { Grottes marines et surplombs sublittoraux à } \\
\text { Corallinales encroûtantes }\end{array}$ & présence & A3.716 & P20.02.06 \\
\hline $\begin{array}{l}\text { Sables grossiers et graviers sublittoraux en } \\
\text { milieux à salinité variable }\end{array}$ & 409 & A5.12 & M08.02 \\
\hline Sables grossiers et graviers infralittoraux & 1060 & A5.13 & M08.01.01 \\
\hline $\begin{array}{l}\text { Sables fins à moyens sublittoraux en milieu à } \\
\text { salinité variable }\end{array}$ & 12219 & A5.22 & M09.03 \\
\hline $\begin{array}{l}\text { Sables mobiles infralittoraux en milieu à salinité } \\
\text { variable à Neomysis integer et Gammarus } \\
\text { spp. }\end{array}$ & - & A5.223 & M09.03.03 \\
\hline $\begin{array}{l}\text { Sables fins propres ou envasés infralittoraux } \\
\text { Sables mobiles propres infralittoraux à faune } \\
\quad \text { éparse } \\
\text { Sables fins infralittoraux à Angulus tenuis } \\
\text { Sables fins infralittoraux à Chamelea striatula - } \\
\quad \text { Mactra stultorum }\end{array}$ & $\begin{array}{c}14229 \\
- \\
- \\
-\end{array}$ & $\begin{array}{c}\text { A5.23 } \\
\text { A5.231 } \\
\text { A5.23 } \\
\text { A5.23 }\end{array}$ & $\begin{array}{c}\text { M09.02.01 } \\
\text { M09.01.01.01 } \\
\text { M09.02.01.01.03 } \\
\text { M09.02.01.01.04 }\end{array}$ \\
\hline $\begin{array}{l}\text { Sables envasés infralittoraux } \\
\text { Sables fins légèrement envasés à Echinocardium } \\
\text { cordatum et Ensis spp. }\end{array}$ & 281 & $\begin{array}{l}\text { A5.24 } \\
\text { A5.241 }\end{array}$ & $\begin{array}{c}\text { M09.02.01.02 } \\
\text { M09.02.01.02.01 }\end{array}$ \\
\hline $\begin{array}{l}\text { Vases infralittorales en milieu à salinité variable } \\
\text { estuarien }\end{array}$ & 26137 & A5.32 & M10.03.02 \\
\hline Vases fluides mobiles infralittorales & - & A5.324 & M10.03.02.04 \\
\hline $\begin{array}{l}\text { Sédiments envasés infralittoraux en milieu à } \\
\text { salinité réduite à Limnodrilus hoffmeisteri, } \\
\text { Tubifex tubifex et Gammarus spp. }\end{array}$ & - & A5.327 & M10.03.02.07 \\
\hline $\begin{array}{l}\text { Sédiments hétérogènes sublittoraux en milieu à } \\
\text { salinité variable }\end{array}$ & 408 & A5.42 & M11.02 \\
\hline
\end{tabular}




\section{XVèmes Journées Nationales Génie Côtier - Génie Civil La Rochelle, 29 au 31 mai 2018}

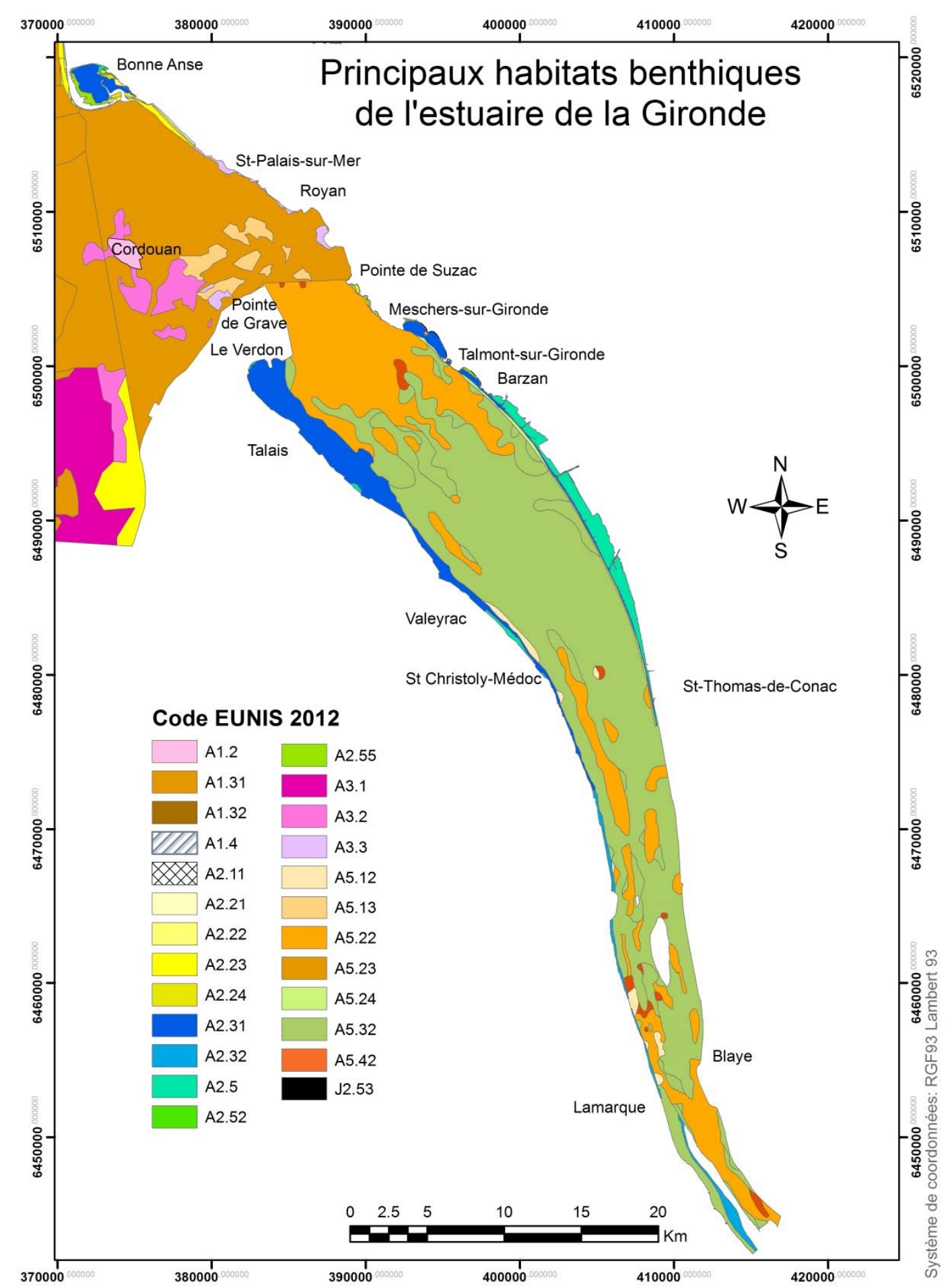

Figure 1. Carte des principaux habitats benthiques de l'estuaire interne et externe de la Gironde selon la typologie EUNIS 2012 (voir les tableaux 1 et 2 pour les codes). Sources CARTHAM, IGN, SHOM, réalisation SAURIAU P.-G. \& BLANCHET H. 


\section{Thème 6 - Gestion durable des zones littorales et estuariennes}

\section{Références bibliographiques}

ACOLAS M.L., LE PICHON C. \& ROCHARD E. (2017). Spring habitat use by stocked one year old European sturgeon Acipenser sturio in the freshwater-oligohaline area of the Gironde estuary. Estuarine, Coastal and Shelf Science, Vol. 196, pp 58-69. https://doi.org/10.1016/j.ecss.2017.06.029

ALLEN G.P., BONNEFILLE R., COURTOIS G. \& MIGNIOT C. (1974). Processus de sédimentation des vases dans l'estuaire de la Gironde. Contribution d'un traceur radioactif pour l'étude du déplacement des vases. La Houille Blanche, Vol. 1/2, pp 129136. https://doi.org/10.1051//hb/1974013

BACHELET G. (1979). Les peuplements macrobenthiques de l'estuaire de la Gironde : évolution annuelle des paramètres démographiques. Journal de Recherche Océanographique, Vol. 4, pp 3-13.

BACHELET G. \& LECONTE M. (2016). Macrofaune benthique. In : Quintin J.Y. (coord.) et al. (2016) : Surveillance écologique du site du Blayais, Rapport scientifique année 2015. In: Rapport IFREMER RST ODE/DYNECO/AG/16-04 (ed. by J.-Y. Quintin, A. Sottolichio, H. Derriennic, S. Doremus, T. Agion, S. Peset, H.D. Hervio, B. Sautour, R. Parra, A. Nowaczyck, G. Bachelet \& M. Leconte), pp. 203-232. Ifremer, Brest. Disponible en ligne sur URL : http://archimer.ifremer.fr/doc/00327/43800/

BAFFREAU A., PEZY J.-P., DANCIÉ C., CHOUQUET B., HACQUEBART P., POISSON E., FOVEAU A., JONCOURT Y., DUHAMEL S., NAVON M., MARMIN S. \& DAUVIN J.-C. (2017). Mapping benthic communities: an indispensable tool for the preservation and the management of the eco-socio-system in the Bay of Seine. Regional Studies in Marine Sciences, Vol. 9, pp 162-173. https://doi.org/10.1016/j.rsma.2016.12.005

BLANCHET H., GOUILLIEUX B., ALIZIER S., AMOUROUX J.-M., BACHELET G., BARILlE A.-L., DAUVIN J.-C., DE MONTAUDOUIN X., DEROLEZ V., DESROY N., GRALL J., GREMARE A., HACQUEBART P., JOURDE J., LABRUNE C., LAVESQUE N., MEIRLAND A., NEBOUT T., OLIVIER F., PELAPRAT C., RUELLET T., SAURIAU P.-G. \& THORIN S. (2014). Multiscale patterns in the diversity and organization of benthic intertidal fauna among French Atlantic estuaries. Journal of Sea Research, Vol. 90, pp 95-110. https://doi.org/10.1016/.jseares.2014.02.014 BOUCHET J.-M. \& LISSALDE J.-P. (1976). Estuaire de la Gironde, étude écologique générale. Macrobenthos et meiobenthos. Rapport de contrat Université de Bordeaux CNEXO N75/1205, Disponible en ligne sur UR1_ : http://archimer.ifremer.fr/doc/00035/14591/

CHARLES N. (2012). Curiosités géologiques de l'Aunis et de la Saintonge, BRGM éditions \& Editions Apogée, Orléans, 111 p.

DOXARAN D., FROIDEFOND J.-M., CASTAING P. \& BABIN M. (2009). Dynamics of the turbidity maximum zone in a macrotidal estuary (the Gironde, France): Observations from field and MODIS satellite data. Estuarine, Coastal and Shelf Science, Vol. 81, pp 321-332. https://doi.org/10.1016/j.ecss.2008.11.013 


\section{XVèmes Journées Nationales Génie Côtier - Génie Civil La Rochelle, 29 au 31 mai 2018}

ESTÈVE G. \& LAHONDÈRE C. (1979). Dossier documentaire : protéger Bonne Anse. Bulletin de la Société Botanique du Centre-Ouest, Nouvelle Série, Vol. 10, pp 71-110. FAÇON R. (1965). La pointe de la Coubre (Charente-Maritime). Étude morphologique. Norois, Vol. 46, pp 165-180.

FROIDEFOND J.-M., JEGOU A.-M., HERMIDA J., LAZURE P. \& CASTAING P. (1998). Variabilité du panache turbide de la Gironde par télédétection. Effets des facteurs climatiques. Oceanologica Acta, Vol. 21, pp 191-207. https://oi.org/10.1016/S03991784(98)80008-X

GEREA (2016a). Inventaires des estrans vaseux et sablo-vaseux de l'estuaire de la Gironde. Disponible en ligne sur UR1_: https://ftp.eau-adour-garonne.fr:8080/sharing/h01ih1nK6

GEREA (2016b). Inventaires des estrans vaseux et sablo-vaseux de l'estuaire de la Gironde. Atlas cartographique, Document final (Planche A3 sur Fond IGN 1/25 000). Disponible en ligne sur URL : https://ftp.eau-adour-garonne.fr:8080/sharing/a9pB60pvB

GUYONNEAU S. (2010). Inventaire raisonné de la malacofaune du plateau de Cordouan (Gironde, France). Bulletin de la Société Linnéenne de Bordeaux, Vol. 145 Nouvelle Série, pp 293-316.

HAMDI A., VASQUEZ M. \& POPULUS J. (2010). Cartographie des habitats physiques Eunis - Côtes de France. Convention Ifremer/AAMP $n^{\circ}$ 09/12177764/FY. Disponible en ligne sur URL : http://archimer.ifremer.fr/doc/00026/13751/

LABAT F. \& SERRETTE D. (2015). Note de chasse dans une mare de tonne à talais (Gironde) : nouveaux taxons pour la faune de France et pour l'Aquitaine. Bulletin de la Société Linnéenne de Bordeaux, Vol. 150 N.S. 43, pp 3-12.

LAHONDÈRE C. (1996). L'estuaire de la Gironde de Royan à Mortagne. Bulletin de la Société Botanique du Centre-Ouest, Nouvelle Série, Vol. 27, pp 393-404.

LAHONDÈRE C. (2002). Contribution à l'étude de la flore et de la végétation de la partie occidentale de Bonne Anse, commune des Mathes (Charente-Maritime). Compte rendu des sorties du 24 juin et du 23 septembre 2001. Bulletin de la Société Botanique du Centre-Ouest, Nouvelle Série, Vol. 33, pp 215-222.

MICHEZ N., BAJJOUK T., AISH A., ANDERSEN A., AR GALL E., BAFFREAU A., BLANCHET H., CHAUVET P., DAUVIN J.-C., DE CASAMAJOR M.-N., DERRIEN-COURTEL S., DUBOIS S., FABRI M.-C., HOUBIN C., LEGALL L., MENOT L., ROLLET C., SAURIAU P.-G., THIÉBAUlT E., TOUROLlE J. \& VAN DEN BELD I. (2015). Typologie des habitats marins benthiques de la Manche, de la Mer du Nord et de l'Atlantique : Version 2. Service du Patrimoine Naturel, Muséum national d'Histoire naturelle, Paris. Disponible en ligne sur URL : https://inpn.mnhn.fr/programme/referentiel-habitats/referentiels-habitats-ou-vegetations

PIGEOT J. (2013). Biodiversité des organismes benthiques du platier rocheux de Cordouan. Annales de la Société des Sciences Naturelles de la Charente-Maritime, Vol. 10, pp 381-412. 


\section{Thème 6 - Gestion durable des zones littorales et estuariennes}

QUENNESON A. \& CASTAGNÉ H. (2012). Plan de conservation des berges à angélique des estuaires. Rapport général. Audenge. Disponible en ligne sur URL : http://www.angeliquedesestuaires.fr/fichiers/realisation.html

SORBE J.C. (1981). La macrofaune vagile de l'estuaire de la Gironde : distribution et migration des espèces, modes de reproduction, régimes alimentaires. Oceanis, Vol. 6, pp 579-592.

SOTTOLICHIO A. \& CASTAING P. (1999). A synthesis on seasonal dynamics of highly-concentrated structures in the Gironde estuary. Comptes Rendus de l'Académie des Sciences - Series IIA - Earth and Planetary Science, Vol. 329, pp 795-800. https://doi.org/10.1016/S1251-8050(00)88634-6

VOLLETTE J. \& THIRION J.-M. (2011a). Etude d'une population d'arénicoles Arenicola marina (Linné, 1767) (Polychaeta, Arenicolae) de la grande Conche de Royan. Annales de la Société des Sciences Naturelles de la Charente-Maritime, Vol. 10, pp 191-197.

VOLLETTE J. \& THIRION J.-M. (2011b). Inventaire préliminaire de la faune du macrobenthos des estrans rocheux calcaires de l'estuaire de la Gironde. Annales de la Société des Sciences Naturelles de la Charente-Maritime, Vol. 10, pp 183-189.

VOLLETTE J. \& THIRION J.-M. (2015). Inventaire de la faune des estrans rocheux calcaires de l'estuaire de la Gironde. Annales de la Société des Sciences Naturelles de la Charente-Maritime, Vol. 10, pp 639-650.

VOLLETTE J., THIRION J.-M. \& LAHONDÈRE C. (2016). Inventaire des macroalgues des estrans rocheux de l'estuaire de la Gironde. Bulletin de la Société Botanique du Centre-Ouest, Nouvelle Série, Vol. 46, pp 25-33. 